\title{
Postpubertal decrease in hippocampal dendritic spines of female rats
}

\author{
Murat Yildirim $^{\text {a,b }}$, Oni M. Mapp ${ }^{\text {b }}$, William G.M. Janssen ${ }^{\text {b, }}$ \\ Weiling Yin ${ }^{\mathrm{c}}$, John H. Morrison ${ }^{\mathrm{b}}$, Andrea C. Gore ${ }^{\mathrm{b}, \mathrm{c}, *}$ \\ a Department of Pharmacology, Hacettepe University, Faculty of Medicine, Ankara, 06100, Turkey \\ ${ }^{\mathrm{b}}$ Department of Neuroscience, Mount Sinai School of Medicine, New York, NY, 10029, USA \\ ${ }^{c}$ Division of Pharmacology and Toxicology, Institute for Neuroscience, and Institute for Cell and Molecular Biology, \\ The University of Texas at Austin, Austin, TX, 78712, USA
}

Received 19 September 2007; revised 7 November 2007; accepted 8 November 2007

Available online 21 November 2007

\begin{abstract}
Hippocampal dendritic spine and synapse numbers in female rats vary across the estrous cycle and following experimental manipulation of hormone levels in adulthood. Based on behavioral studies demonstrating that learning patterns are altered following puberty, we hypothesized that dendritic spine number in rat hippocampal CA1 region would change postpubertally. Female Sprague-Dawley rats were divided into prepubertal (postnatal day (P) 22), peripubertal (P35) and postpubertal (P49) groups, with the progression of puberty evaluated by vaginal opening, and estrous cyclicity subsequently assessed by daily vaginal smears. Spinophilin immunoreactivity in dendritic spines was used as an index of spinogenesis in area CA1 stratum radiatum (CA1sr) of hippocampus. First, electron microscopy analyses confirmed the presence of spinophilin specifically in dendritic spines of CA1sr, supporting spinophilin as a reliable marker of hippocampal spines in young female rats. Second, stereologic analysis was performed to assess the total number of spinophilin-immunoreactive puncta (i.e. spines) and CA1sr volume in developing rats. Our results indicated that the number of spinophilin-immunoreactive spines in CA1sr was decreased $46 \%$ in the postpubertal group compared to the two younger groups, whereas the volume of the hippocampus underwent an overall increase during this same developmental time frame. Third, to determine a potential role of estradiol in this process, an additional group of rats was ovariectomized (OVX) prepubertally at P22, then treated with estradiol or vehicle at $\mathrm{P} 35$, and spinophilin quantified as above in rats perfused on P49. No difference in spinophilin puncta number was found in OVX rats between the two hormone groups, suggesting that this developmental decrease is independent of peripheral estradiol. These changes in spine density coincident with puberty may be related to altered hippocampal plasticity and synaptic consolidation at this phase of maturity.
\end{abstract}

(C) 2007 Elsevier Inc. All rights reserved.

Keywords: Puberty; Dendritic spine; Hippocampus; CA1; Spinophilin; Estrogen; Synaptic plasticity; Spinogenesis

\section{Introduction}

In humans, puberty is associated with alterations in cognitive ability and style. For example, ease in new language acquisition or the ability to learn a musical instrument is high prepubertally and declines thereafter (reviewed in Komarova and Nowak, 2001; Yun et al., 2004). Pubertal changes in cognitive function are also reported in rodents, in which alterations in spatial learning occur across the pubertal period (Kanit et al., 2000; Schenk, 1985).

\footnotetext{
* Corresponding author. The University of Texas at Austin, Division of Pharmacology and Toxicology, A1915, Austin, TX, 78712, USA. Fax: +1 512 4715002.

E-mail address: andrea.gore@mail.utexas.edu (A.C. Gore).
}

Although the mechanisms for these alterations in cognitive function are poorly understood, they may be due to developmental changes in synaptic plasticity that occur across the pubertal period. Consistent with this, reports show that synaptic density and/ or dendritic spine number decrease during the transition from puberty to adulthood, as shown in monkey cortex (somatosensory: Zecevic and Rakic, 1991; prefrontal: Woo et al., 1997; visual: Bourgeois and Rakic, 1993) hamster medial amygdala (Zehr et al., 2006), and mouse hippocampus (Meyer et al., 1978).

Along with developmental age, hormones such as estrogen regulate synaptic plasticity in the hippocampus of female rodents (Gould et al., 1990; Woolley and McEwen, 1992; McCarthy and Milner, 2003; Woolley et al., 1990). During the natural fluctuations in sex steroid hormones that occur across estrous cycles 
of rats, dendritic spine density in hippocampal CA1 pyramidal neurons decreases on days of low estrogen, e.g. estrus, compared to days of high estrogen levels, e.g. proestrus (Gould et al., 1990; Woolley and McEwen, 1992; McCarthy and Milner, 2003; Woolley et al., 1990). Studies involving experimental manipulation of estrogen levels in ovariectomized adult rats show similar facilitatory effects of estrogen on dendritic spines (Gould et al., 1990; Woolley and McEwen, 1992; McCarthy and Milner, 2003; Bi et al., 2000; Woolley et al., 1996). These changes in estrogen across natural or manipulated reproductive cycles correlate not only with structural synaptic changes but also with behavioral cognitive outcomes (Romeo, 2003; Gibbs, 2000a; Luine et al., 2003).

While effects of age and estrogen and their interactions on cognition and neural plasticity have been studied extensively during reproductive aging (reviewed in Adams and Morrison, 2003), these questions are not well-studied in the context of puberty. This is an important question for several reasons. First, the pubertal period is associated with dramatic changes in circulating hormones, comparable to, if not greater than, (Apter, 1997) changes occurring during reproductive aging (Hammond, 1996). Second, hormone-associated changes in brain plasticity such as neurite outgrowth, spinogenesis, synaptogenesis, and expression of neurotransmitter receptors are observed during puberty in neuroendocrine brain regions (Anderson, 1982; Cottrell et al., 2006; Gore et al., 1996; Matsumoto and Arai, 1976; Reier et al., 1977); however such analyses have not been systematically studied in hippocampal brain regions. Third, puberty is associated with alterations in learning and memory (Kanit et al., 2000; Romeo, 2003; Wright and Zecker, 2004), phenomena that are likely to be related to dendritic spine number and density in hippocampus in adults (Gould et al., 1990; Woolley and McEwen, 1992; Woolley et al., 1990; Woolley et al., 1996). Thus, we predict that both developmental age as well as hormonal changes occurring during puberty will alter synaptic plasticity in the hippocampus.

In the present study, we hypothesized that dendritic spine number in rat hippocampus would change postpubertally, focusing on the CA1 stratum radiatum (CA1sr). This region is particularly sensitive to effects of estrogen or estrous cycle on dendritic spine density and on $N$-methyl-D-aspartate (NMDA) receptor-dependent long-term potentiation (Woolley and McEwen, 1994; Warren et al., 1995; Murphy and Segal, 1996). However, how such changes would be manifested is difficult to predict, because as discussed above, puberty is associated with elevated estrogen levels, suggesting increases in dendritic spines. Moreover, the elevated neurite outgrowth and arborization in hypothalamus in response to estrogens (Reier et al., 1977; Matsumoto and Arai, 1976) also leads to a prediction that such changes may occur in hippocampus. By contrast, as discussed above, puberty is accompanied by decreased synapse number in several brain regions (Zecevic and Rakic, 1991; Bourgeois et al., 1994; Zehr et al., 2006; Meyer et al., 1978). To address these issues, using high-resolution microscopy we quantified spinophilin immunoreactivity in CA1sr as a marker of dendritic spines (Hao et al., 2003; Tang et al., 2004) in female rats during the pubertal process. We also investigated the estrogen-dependence of this process using an ovariectomy model, comparing rats given estradiol or vehicle treatment.

\section{Materials and methods}

\section{Animals}

All animal protocols were approved by the Institutional Animal Care and Use Committee of Mount Sinai School of Medicine, following guidelines provided in The Guide for the Care and Use of Laboratory Animals. A total of 34 female Sprague-Dawley rats were housed 2-3 per cage in a temperaturecontrolled room $\left(22{ }^{\circ} \mathrm{C}\right)$ with a 12-hour-light and 12-hour-dark cycle (lights on $07.00 \mathrm{~h}$ ). Food and water were available $a d$ libitum.

\section{Experiment 1: electron microscopy}

In order to confirm the dendritic localization of spinophilin in the CA1sr of hippocampus of young female rats, four intact postpubertal female rats at postnatal day (P) 49 were anesthetized and euthanized as described below.

\section{Experiment 2: light microscopy - intact rats}

Rats were assigned to one of three age groups: prepubertal (P22, $n=6)$, pubertal (P35, $n=6)$ and postpubertal (P49, $n=5)$ groups. A subset of rats was euthanized on P22 (prepubertal). For the remaining rats, the progression of puberty was evaluated by daily observation until the occurrence of vaginal opening (Gore et al., 1996; Daftary and Gore, 2003), a marker of reproductive maturation in rodents (Safranski et al., 1993), which occurred on P35 in this study. A subset of rats was euthanized at P35 (pubertal). The remaining rats were subjected to daily vaginal smears, and euthanized on P49 (postpubertal). Although postpubertal rats on $\mathrm{P} 49$ were at different stages of the estrous cycle at euthanasia, this did not correlate with any differences in spinophilin immunoreactivity. Therefore data presentation represents group data from randomly cycling rats at the postpubertal stage.

\section{Experiment 3: light microscopy - OVX rats}

Rats were bilaterally ovariectomized (OVX) under halothane anesthesia at P22. Two weeks later (P35), rats were again anesthetized with halothane, and implanted subcutaneously with a Silastic capsule $(1 \mathrm{~cm}$ length, inner diameter $1.96 \mathrm{~mm}$, outer diameter $3.18 \mathrm{~mm}$ ) containing either $10 \%$ estradiol $/ 90 \%$ cholesterol (E2 group, $n=7$ ) or the vehicle (VEH group, 100\% cholesterol, $n=6$ ). Two weeks later (P49), rats were euthanized. The carcasses were examined for the complete removal of the ovaries, and the efficacy of the estrogen treatment on the uterus. Rats treated with E2 had large, swollen uteri, demonstrating a uterotrophic effect of estrogen, while VEH rats had atrophied uteri (Gore et al., 2002).

\section{Euthanasia and perfusion}

Rats were deeply anesthetized with $30 \%$ chloral hydrate and perfused transcardially with ice-cold $1 \%$ paraformaldehyde in 
phosphate buffered saline (PBS) for $1 \mathrm{~min}(50 \mathrm{ml} / \mathrm{min})$ followed by cold $4 \%$ paraformaldehyde $/ 0.125 \%$ glutaraldehyde in PBS for $12 \mathrm{~min}$ (Adams et al., 2002). All perfusions took place between 1200 and $1400 \mathrm{~h}$. The brains were removed from the skull and postfixed overnight in the same fixative at $4{ }^{\circ} \mathrm{C}$. Fifty micrometer thick sections were cut on a vibrating microtome the following day and sections stored in PBS.

\section{Immunohistochemistry}

Sections were thoroughly rinsed in $0.01 \mathrm{M}$ PBS containing $0.3 \%$ Triton X-100 (Sigma, St. Louise, MO), and incubated in blocking buffer containing $0.3 \%$ Triton X-100, $0.1 \%$ cold water fish gelatin (Electron Microscopy Sciences [EMS], Fort Washington, PA), $0.5 \%$ bovine serum albumin (Sigma, St. Louis, MO), and 5\% normal goat serum (Vector Laboratories, Burlingame, CA) for $1 \mathrm{~h}$ at room temperature. As described previously (Hao et al., 2003; Tang et al., 2004), free-floating sections were incubated with a well-characterized and highly specific rabbit anti-spinophilin primary antibody (kindly provided by P.B. Allen and P. Greengard; Allen et al., 1997; Feng et al., 2000), diluted 1:240,000 in above blocking buffer for 5 days at $4{ }^{\circ} \mathrm{C}$, washed, and incubated in secondary antibody (goat anti-rabbit $\mathrm{IgG}$, ultra small electron microscopy grade, EMS) in the above diluents overnight at room temperature. Sections were washed and postfixed with $2 \%$ glutaraldehyde in PBS, and rinsed with distilled water. Silver enhancement was performed with Auron R-Gent-LM kit (EMS) for 15-20 min at room temperature. After washing, the sections were mounted on gelatin-subbed slides, dehydrated through ascending series of ethanol, and coverslipped with Biomount (EMS). In control experiments the primary antibody was omitted, and no immunostaining was observed. Spinophilin immunohistochemistry was quantified on sections containing the CA1 region of hippocampus. Here, dendritic spines were identified immunohistochemically as small puncta labeled with anti-spinophilin and quantified by an unbiased stereologic methodology described below.

\section{Stereologic analysis}

A series of every 10th section through the entire rostro-caudal extent of the hippocampus of each animal was used in this study, which resulted in an average of 8 sections per series. The sections from each animal were coded before the quantitative analysis, and the code was not broken until after the analysis was complete. The optical fractionator (West et al., 1991), a stereologic method, was used to estimate the total number of spines in CA1sr. This method provides an unbiased estimate of spine number, and it relies on random, systematic sampling from a known fraction of a structure's total volume (West et al., 1991; Bjugn, 1993). All quantitative analyses were performed using a computer-assisted morphometry system consisting of a Zeiss Axioplan 2 photomicroscope equipped with an Applied Scientific Instrumentation MS-2000 XYZ computer-controlled motorized stage, a DAGE-MTI DC-330 video camera, a Gateway microcomputer, and the Stereo Investigator morphometry and stereology software (MicroBrightField version 5, Williston, VT). All these procedures have been described previously and are routine within the laboratories (Hao et al., 2003; Tang et al., 2004; Chakraborty et al., 2003a,b). The contours of CA1sr were traced at $2.5 \times$ magnification. Optical disector counting frames were placed in a systematic random fashion in the delineated regions of the sections with constant intervals in the $x$ - and $y$-axes. The counting frame width and height was set at $1.5 \mu \mathrm{m}$, the $z$-axis thickness of the counting frame was $3 \mu \mathrm{m}$ and the grid size was $250 \mu \mathrm{m} \times 250 \mu \mathrm{m}$. The area sampling fraction was $(1.5 \times 1.5) /(250 \times 250)=9 / 250,000$. A Zeiss oil-immersion objective $(100 \times / 1.4 \mathrm{NA})$ was used for counting. A $2 \mu \mathrm{m}$ "guard zone" was put at the top surface of the sections. Counting was performed with the optical disector technique through a depth of $3 \mu \mathrm{m}$ (the height of the disector). The total spinophilin-immunoreactive spine number in each area was calculated by using standard stereologic formulas that are built into the optical fractionator protocols in MicroBrightField. The total volume of each analyzed layer of CA1 was estimated using the Cavalieri principle (Gundersen et al., 1988) to assess whether changes in the total number of spines might be due to changes in the volume of each area or changes in the spine density.

\section{Pre-embedding electron microscopy (EM)}

Silver-enhanced nanogold localization of spinophilin was performed using cryosubstitution EM in order to assess the suitability of spinophilin as a spine marker in rat hippocampus. For this purpose, a different set $(n=4)$ of $\mathrm{P} 49$ postpubertal rats was used, and they were perfused and tissues processed identically to the previous set of rats. Following immunocytochemistry with the spinophilin antibody, freeze substitution and low temperature embedding of the specimens was performed as described previously (Van Lookeren Campagne et al., 1991; Adams et al., 2001). In brief, tissues were cryoprotected by immersion in increasing concentrations of glycerol in phosphate buffer $(10,20$, and $30 \%$ ) and were plunged rapidly into liquid propane cooled by liquid nitrogen $\left(-190{ }^{\circ} \mathrm{C}\right)$ in a Universal Cryofixation KF80 (Reichert-Jung, Vienna, Austria). The samples were immersed in $1.5 \%$ uranyl acetate (for en bloc fixation) in anhydrous methanol $\left(-90{ }^{\circ} \mathrm{C}, 24 \mathrm{~h}\right)$ in a cryosubstitution Automated Freeze Substitution unit (Leica, Vienna, Austria). The temperature increased in steps of $4{ }^{\circ} \mathrm{C} / \mathrm{h}$ from $-90{ }^{\circ} \mathrm{C}$ to $-45{ }^{\circ} \mathrm{C}$. The samples were washed with anhydrous methanol and infiltrated with Lowicryl $\mathrm{HM} 20$ resin (EMS) at $-45^{\circ} \mathrm{C}$ with a progressive increase in the ratio of resin to methanol for $2 \mathrm{~h}$ each, followed with pure Lowicryl (overnight). Polymerization was performed with ultraviolet light $(360 \mathrm{~nm})$ at $-45{ }^{\circ} \mathrm{C}$ for $48 \mathrm{~h}$, followed by $24 \mathrm{~h}$ at room temperature. The stratum radiatum of the CA1 region (about $150-200 \mu \mathrm{m}$ from the cell bodies) was identified and sectioned. Serial ultrathin sections $(5 \times 70-80 \mathrm{~nm}$ thick $)$ were cut with a diamond knife on a Reichert-Jung ultramicrotome and mounted on formvar coated slot grids for analysis. A synapse was considered immunoreactive if it contained silver-intensified product in any individual section within the series of five sections. The sections were analyzed on a JEOL 1200EX electron microscope (JEOL, Tokyo, Japan). Images were captured using a 
high-resolution Advantage CCD camera (Advanced Microscopy Techniques Corporation, Danvers, MA). Control experiments omitting the primary antibody were performed, and no labeling was observed.

\section{Estradiol radioimmunoassay}

Estradiol concentrations in serum were estimated by radioimmunoassay (Diagnostic Systems Laboratory, Inc, Webster, TX) as described previously (Gore et al., 2002). Serum samples $(200 \mu$ l) from each rat in Experiment 2 (intact rats at P22, 35 and 49) were assayed in duplicate in a single assay. Intra-assay coefficient of variation was $5.0 \%$ and assay sensitivity was $5 \mathrm{pg} /$ $\mathrm{ml}$. Unfortunately, due to a technical error, blood samples from Experiment 3 (OVX plus E2 or VEH treatment, P49) were lost so we were unable to confirm concentrations of estradiol. However, all OVX + VEH rats had atrophied uteri, whereas all $\mathrm{OVX}+\mathrm{E} 2$ rats had swollen uteri, confirming efficacy of estradiol treatment (Gore et al., 2002). In addition, post-mortem analysis confirmed that all ovaries were fully removed in all surgically OVX rats.

\section{Statistical analysis}

For the stereologic analysis, the coefficients of variation $(\mathrm{CV}=$ standard deviation / mean) and coefficient of error (CE) of the stereologic estimates were calculated as previously described 'Tang et al., 2004; Chakraborty et al., 2003a,b). For each animal, CE's ranged from 0.05 to 0.12 , and mean CE's were 0.07 to 0.09 per group. Statistical analysis of results in Experiment 1 was performed with one-way ANOVA (variable: age) followed by post-hoc analyses when a significant main effect was determined. For Experiment 2, differences between $\mathrm{OVX}+\mathrm{E} 2$ and $\mathrm{OVX}+\mathrm{VEH}$ rats were determined by $t$-test. In both cases, effects were considered significant at $p<0.05$. All values are given as means \pm SEM. Photomicrographs were captured and further processed by using the Adobe Photoshop 7.0 program. Only minor adjustments of brightness and contrast were made as described in previous studies from our laboratory (Chakraborty et al., 2003b), and these adjustments in no way altered the appearance of the original material.

\section{Results}

Experiment 1: electron microscopic analysis of spinophilin localization in CAlsr dendritic spines

Electron microscopy was used to confirm the specific subcellular localization of spinophilin within dendritic spines of hippocampal CA1sr from four intact female rats at P49. Spinophilin immunoreactivity was distributed within and throughout axospinous synapses (Fig. 1). Multiple silver-intensified particles
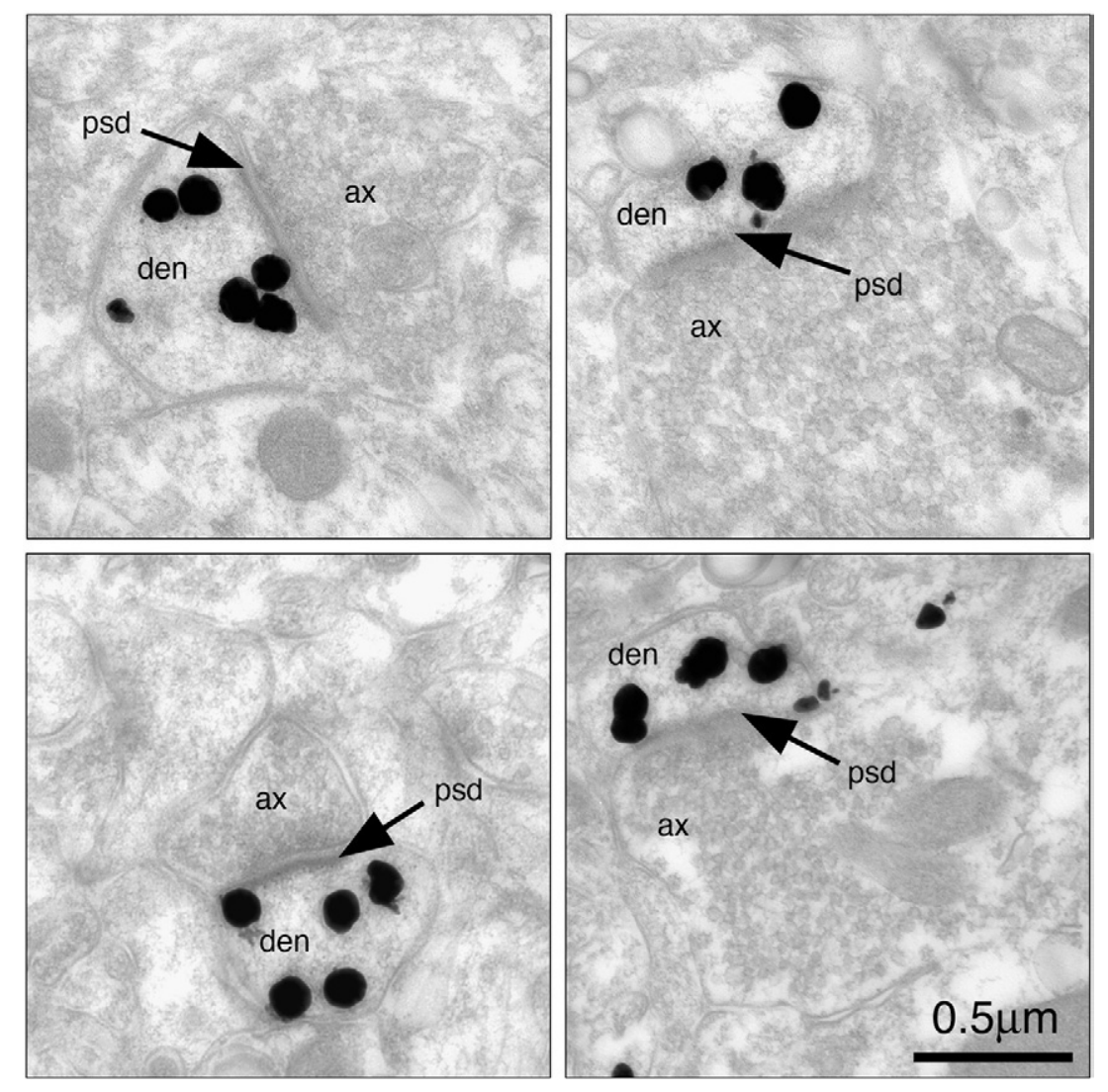

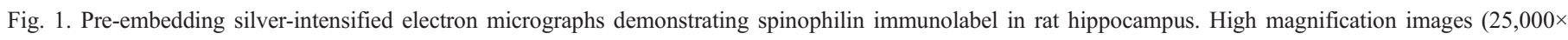



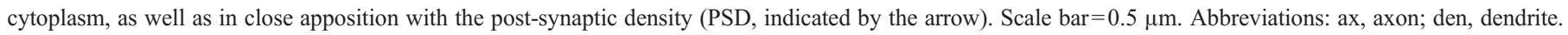




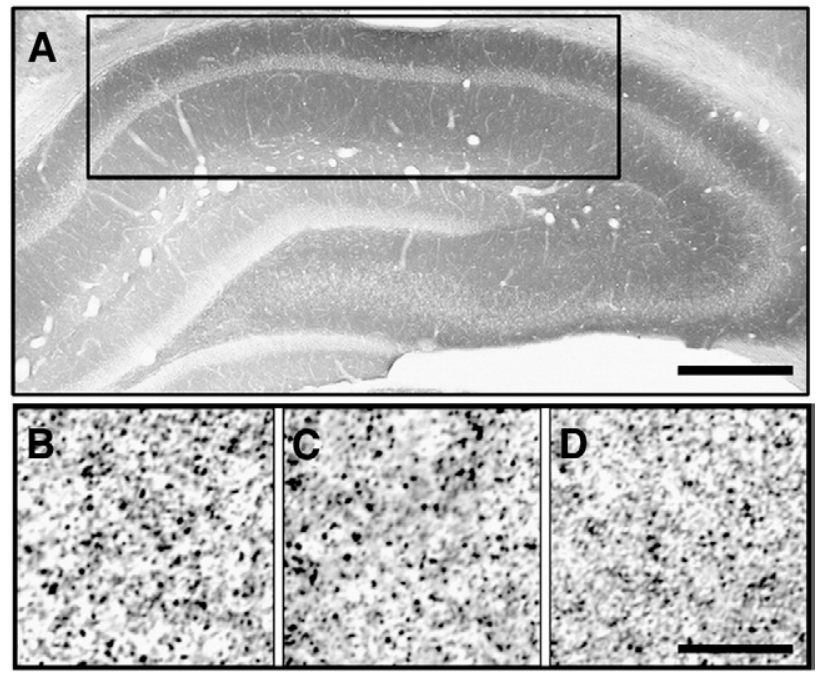

Fig. 2. Light micrographs of spinophilin-labeled puncta in hippocampal CA1sr of intact female rats. Panel A is a low-power photomicrograph of spinophilin immunoreactivity in a representative $\mathrm{P} 35$ rat. The black rectangular box represents the CA1 region of the hippocampus, where analyses were performed. High-power (digitally cropped and enlarged for demonstration of spinophilin-immunoreactive puncta) photomicrographs are shown in panels B-D for a representative prepubertal (P22), pubertal (P35), and postpubertal (P49) rat, respectively. Scale bars: $\mathrm{A}=900 \mu \mathrm{m}, \mathrm{D}=15 \mu \mathrm{m}$.

were seen throughout the cytoplasm, as well as in close apposition with the post-synaptic density. The majority of axospinous synapses were spinophilin immunopositive. Single section (2dimensional) analysis consistently demonstrated that approximately $70 \%$ of axospinous synapses in CA1sr were spinophilin immunoreactive. Serial analysis significantly increased the percentage of spinophilin-immunoreactive labeled synapses to approximately $89 \%$, thus establishing spinophilin as a reliable marker for dendritic spines in CA1sr of rats.



Fig. 3. Comparison of the total spinophilin-immunoreactive puncta numbers $\left(\times 10^{9}\right)$ in hippocampal CA1sr of prepubertal, pubertal and postpubertal rats. A significant age-related decrease $(46 \%)$ was detected $(p<0.05)$. Although there was no statistical difference between the prepubertal and pubertal groups, postpubertal rats had significantly lower puncta numbers than rats in the two younger ages. $*, p<0.05$.

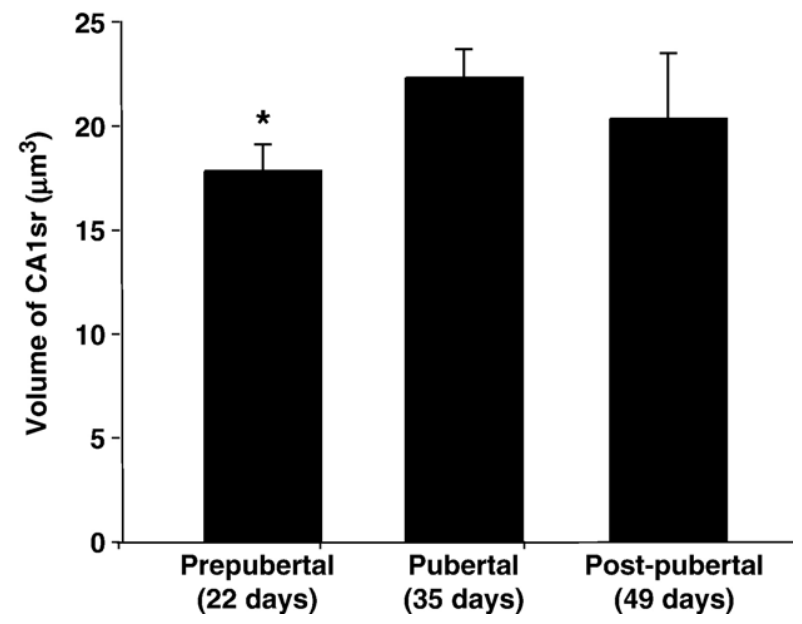

Fig. 4. Comparison of the volumes $\left(\mu \mathrm{m}^{3}\right)$ of the hippocampal CA1sr of prepubertal, pubertal and postpubertal groups. Overall a significant age-related increase in CA1sr volume was determined $(p<0.05)$. Prepubertal female rats had a significantly smaller CA1sr volume $(20 \%)$ than pubertal rats. ${ }^{*}, p<0.05$.

\section{Experiment 2: quantification of spinophilin-labeled puncta in CAlsr of intact female rats}

Spinophilin immunoreactivity was localized throughout the hippocampus, including the CA1sr region. Fig. 2A shows a lowpower photomicrograph of spinophilin immunoreactivity in a representative rat. High-power photomicrographs are shown in Fig. 2B-D for a representative prepubertal, pubertal, and postpubertal rat, respectively. Stereologic analysis of spinophilinimmunoreactive puncta in CA1sr of rats indicated significant differences among the three age groups $(p<0.05$; Fig. 3$)$. We detected similar numbers of puncta in prepubertal and pubertal rats, but a significant (46\%) decrease in postpubertal rats (Fig. 3). These differences between the postpubertal group and the prepubertal and pubertal groups were statistically significant $(p<0.05)$.

The volume of the CA1sr region was calculated using the MicroBrightField program. Statistical analysis of these results demonstrated significant differences in CA1sr volume with developmental age $(p<0.05$; Fig. 4). Further post-hoc analysis demonstrated that CA1sr volume was significantly different between prepubertal $(\mathrm{P} 22)$ and pubertal $(\mathrm{P} 35)$ rats $(p<0.05)$, with sizes approximately $20 \%$ smaller in the prepubertal group (Fig. 4). However, this difference was not continued throughout the postpubertal group, in which volume was on average $\sim 10 \%$

Table 1

Serum estradiol concentrations and body weight in developing intact female Sprague-Dawley rats (Experiment 2)

\begin{tabular}{lcc}
\hline Age & Serum estradiol $(\mathrm{pg} / \mathrm{ml})$ & Body weight $(\mathrm{g})$ \\
\hline Prepubertal (P22) & $9.0 \pm 1.6$ & $39.2 \pm 0.8$ \\
Pubertal (P35) & $26.0 \pm 3.9^{*}$ & $92.3 \pm 1.2^{*}$ \\
Postpubertal (P49) & $33.9 \pm 9.8^{*}$ & $103.6 \pm 0.8^{*}$ \\
\hline
\end{tabular}

Group data are shown for rats on the day of euthanasia (between 1200 and $1400 \mathrm{~h}$ ) as mean \pm SEM. * $p<0.05$ vs. prepubertal. $\mathrm{P}=$ postnatal days of age. 




Fig. 5. Effects of estradiol (E2) or vehicle (VEH) on spinophilin-immunoreactive puncta numbers $\left(\times 10^{9}\right)$ in hippocampal CA1sr of 49 day-old female rats that were OVX prepubertally at P22, given a Silastic capsule containing E2 or VEH pubertally at P35, and euthanized at P49. No difference in spinophilin puncta numbers was detected by stereologic analyses.

higher in postpubertal than pubertal rats $(p>0.05)$, possibly due to greater variability of CA1sr volume in the postpubertal rats. The average body weights of the rats in the prepubertal, pubertal and postpubertal groups were 39, 92 and 103 g respectively (Table 1). Thus, the hippocampal CA $1 \mathrm{sr}$ increases by $20 \%$ from the prepubertal to the pubertal stage, a developmental period when the overall increase in body weight is $235 \%$.

\section{Estradiol concentrations in intact rats}

Serum estradiol concentrations of intact rats were assayed by radioimmunoassay. The results indicated a significant developmental increase in estradiol concentrations $(p<0.05$; Table 1$)$. Individual differences among animals within the postpubertal age group were attributable to differences in estrous cyclicity. Concentrations at the pubertal and postpubertal ages were significantly higher than those at the prepubertal age $(p<0.05)$.

\section{Experiment 3: quantification of spinophilin-labeled puncta in CA1sr of OVX female rats}

In order to assess the role of estradiol in the developmental change in spinophilin puncta number, rats were OVX prepubertally (P22), given E2 or VEH treatment (P35), and euthanized at P49. As shown in Fig. 5, total spinophilin puncta number did not differ between the two groups, nor did they differ when compared to intact rats at P49 (c.f. Fig. 3). CA1sr volume was also similar between OVX $+\mathrm{VEH}$ and $\mathrm{OVX}+\mathrm{E} 2$ rats at P49 (Fig. 6).

\section{Discussion}

The present study demonstrates a significant change in spinophilin immunoreactivity, a marker of dendritic spine number, in hippocampal CA1 sr during pubertal development in intact female rats. Overall, spinophilin puncta number decreased $46 \%$ in the postpubertal age group compared to prepubertal and pubertal rats.
By contrast, the volume of the CA1sr region increased slightly but significantly with age from the prepubertal to the pubertal stage. Thus, overall dendritic spine density decreased throughout the pubertal process, a phenomenon that may underlie pubertal-associated changes in learning and memory (Krasnoff and Weston, 1976).

Spinophilin is a marker for dendritic spines in developing rat hippocampus

In the present study we utilized spinophilin protein expression as an indicator of dendritic spines in CA1sr of female rats. Spinophilin is a protein phosphatase 1-binding protein that specifically localizes to the heads of dendritic spines (Allen et al., 1997; Feng et al., 2000). It regulates spine formation and function (Allen et al., 1997; Feng et al., 2000) and is responsive to estrogen in the hippocampus of adult rats (Brake et al., 2001). Previous work from one of our laboratories (J.H.M.) has validated the stereologic quantification of spinophilin-immunoreactive puncta using the same antibody in the monkey (Hao et al., 2003), and our present data are consistent with that earlier report. Our electron microscopy findings show the spinophilin label in rat CA1sr region and provide additional ultrastructural information about its subcellular localization. High magnification electron micrographic images show spinophilin distributed within and throughout axospinous synapses. Multiple silver-intensified particles are seen throughout the cytoplasm, as well as in close apposition to the post-synaptic density. We infer that the high particle density within spine heads in electron micrographs is resolved as the discrete, individual puncta that are seen in the light microscopic analysis. Further analysis of serial sections by electron microscopy indicates that approximately $90 \%$ of axospinous synapses are spinophilin immunopositive in hippocampal CA1sr of female rats. The light microscopic analyses confirm robust spinophilin-immunoreactivity expression throughout hippocampal CA1. Thus, we conclude that spinophilin can be used as a reliable marker of

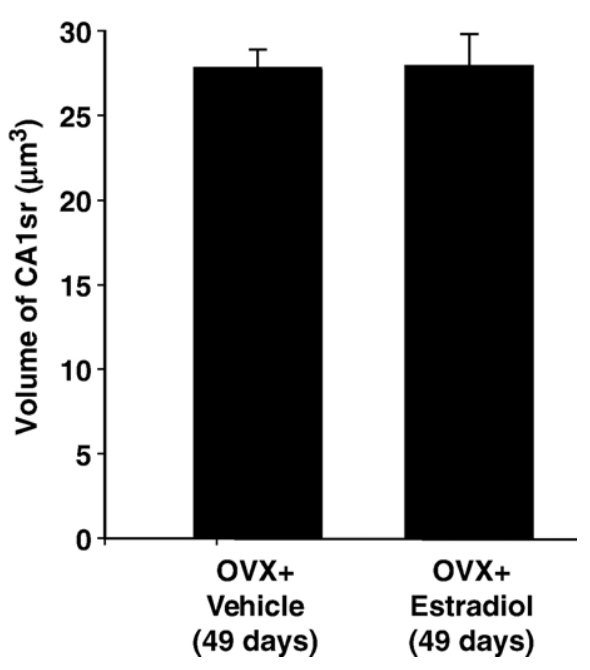

Fig. 6. Comparison of the volumes $\left(\mu \mathrm{m}^{3}\right)$ of the hippocampal CA1sr in P49 rats that were $\mathrm{OVX}+\mathrm{E} 2$ or OVX $+\mathrm{VEH}$ treated as described in Fig. 5. No differences in CA1sr volume were detected. 
dendritic spines in developing female rats, similar to our previous findings in rhesus monkeys (Hao et al., 2003; Tang et al., 2004).

\section{Hippocampal plasticity and puberty}

The peripubertal period of maturation is characterized by activation of the hypothalamic-pituitary-gonadal axis (Gore, 2001). This process begins in the central nervous system with the activation of hypothalamic-preoptic gonadotropin-releasing hormone neurons, continues through the subsequent activation of pituitary gonadotropin release, and is ultimately manifested as a stimulation of the gonad. The subsequent increases in steroidogenesis, including estradiol biosynthesis and gametogenesis, cause secondary sex characteristic development and fertility (reviewed in Gore, 2001). During the pubertal developmental stage, substantial changes occur in the hypothalamus, as reflected by increased synaptogenesis (Matsumoto and Arai, 1977), elevated dendritic spine density (Anderson, 1982), and ultrastructural changes such as organelle density (Reier et al., 1977). However, to our knowledge, the question of whether pubertal changes in hippocampal synaptic organization occur during the pubertal process has only been addressed by two groups. The first report (Meyer et al., 1978) counted dendritic spines in Golgi-impregnated CA1 tissues of male mice, and showed that the number of dendritic spines increased from P25 to P35 (during the progress of puberty), were maintained at this level through P45, and subsequently decreased to P55. A second group (Andersen and Teicher, 2004) used synaptophysin as a marker of synapses, and showed developmental changes in CA1 of female rats, with a decrease from day 80 to 100 . Although not significant, the authors also found a decrease from days 40 to 60 . Differences between that study and ours are probably attributable to the choice of markers, spinophilin in our study, and synaptophysin in that of Andersen and Teicher (2004). These two markers do not exist in the same subcellular compartment (Choi et al., 2003) and in fact, a study by Zehr et al. found opposite pubertal changes in spinophilin (decrease) and synaptophysin (increase) in the amygdala of developing male hamsters (Zehr et al., 2006).

At a gross morphological level, Koshibu et al. showed that total hippocampal volume, estimated by magnetic resonance microscopy, increases during puberty (from one month to three months of age) in male and female C57B1/6 mice (Koshibu et al., 2004). Measurements of changes in size of limbic nuclei of female rats show slight fluctuations during the pubertal period with, in general, an overall increase from P21 to P36 (Docke and Smollich, 1978). Together, these studies show an increase in hippocampal volume during puberty, similar to our present findings. There are also functional behavioral changes during development that are likely to be due at least in part to hippocampal maturation. For example, the hippocampal-dependent task of place navigation, assayed by the Morris water maze, shows a developmental improvement from postnatal days 21 through 64, encompassing the pubertal period (Schenk, 1985). In a different maze task, postpubertal female rats make more errors than prepubertal rats (Krasnoff and Weston, 1976). This literature is suggestive of changes in hippocampal morphology and ultra- structure during the same developmental period when the ability to learn and remember undergoes changes.

Our present studies extend these previous reports by showing a specific change in spinophilin in hippocampal CA1sr of female rats undergoing puberty. We showed that postpubertal rats have significantly lower dendritic spine number and density than prepubertal or pubertal rats. The decrease in dendritic spines in male mice from P45 to P55 reported by Meyer et al. (1978) is consistent with our present observation. Moreover, a recent report by Zehr et al. (2006) showed that spinophilin Protein levels in the amygdala of male hamsters declined about $35-40 \%$ during pubertal maturation, similar to our current observed decrease of about $45 \%$ in CA 1 sr of female rats. Additionally, that study in hamster (Zehr et al., 2006) showed that the pubertal decline in spinophilin protein occurred during a developmental time period during which dendritic pruning occurred in the amygdala. Together with that study, our results support a pubertal decrease in dendritic spines in two brain regions, as indicated by declines in spinophilin protein. In the future we intend to relate our observed changes in dendritic spine numbers to changes in hippocampal-mediated behavioral functions in pubertal rats.

Other laboratories have reported changes in markers of synaptic plasticity associated with pubertal development in a variety of species. Zecevic and Rakic Zecevic and Rakic (1991) demonstrated a decrease in synaptic density in somatosensory cortex of rhesus monkeys during the pubertal period. In their experiment, monkeys were studied that ranged in age from embryonic day 41 to 20 years, and while the density of synapses per unit volume of cortex as well as per unit volume of neuropil increased steadily throughout the late fetal ages and early infancy, at puberty, there was a decrease in the concentration of synapses. This was primarily due to elimination of asymmetrical junctions located on dendritic spines while symmetrical synapses on dendritic shafts and cell bodies remained relatively constant during postnatal life. Another report by Bourgeois et al. (1994) examined the course of synaptogenesis in the prefrontal cortex in rhesus monkeys ranging in age from embryonic day 47 to 20 years of age. They described different phases for synapse number and formation, and found that the rapid phase of synaptogenesis begins at 2 months before birth and ends approximately at 2 months after birth, due largely to a selective increase in axospinous synapses in the supragranular layers. From that point, synaptic density remains relatively constant until about 3 years of age. At that age, rhesus monkeys begin to attain adult reproductive function, and synaptic density begins to show a slight but significant decline in synaptic density through $20+$ years of age. Finally, in another study in monkey prefrontal cortex, Woo et al. (1997) showed a peripubertal elimination of axospinous synapses and dendritic spines, together with topographic refinement, again suggesting that this region undergoes substantial reorganization during late postnatal development. These findings in the monkey cortex suggest that synaptic density increases during embryonic and early postnatal development, but begins to switch to a decrease at puberty. These results are quite similar to our present results in the rat hippocampus. 


\section{Is there a role for estradiol in the pubertal changes in hippocampal dendritic spines?}

Pubertal developmental in female mammals is characterized by dramatic increases in circulating estradiol concentrations (Apter, 1997; see Table 1). This hormone acts on targets throughout the body and the brain, where estrogen receptors are widely expressed (Chakraborty and Gore, 2004; Mitra et al., 2003). Thus, during puberty, there can be estrogen-dependent as well as estrogen-independent developmental changes in brain circuitry (Romeo, 2003). Evidence for the estrogen-dependence of this process is shown in the developing hypothalamus, in which the increases in indicators of brain plasticity are accelerated by administration of exogenous estradiol to prepubertal female rats (Segarra and McEwen, 1991; Clough and Rodriguez-Sierra, 1983). Similarly, in ventromedial hypothalamus, estradiol treatment to ovariectomized rats increases dendritic plasticity (Frankfurt et al., 1990; Carrer and Agustin, 1982). These findings suggest a causal relationship between increasing estradiol levels (either during puberty or to ovariectomized adults) and their subsequent effects on hypothalamic neurons. Although the hypothalamus is the most obvious target to study steroid-induced plasticity, it is becoming increasingly clear that other brain regions, including hippocampus, respond to hormones such as estradiol with increases in dendritic spine number and density (Woolley and McEwen, 1992; Hao et al., 2003) and increased expression of synaptic proteins (Brake et al., 2001). Estrogens alter hippocampal excitability (Scharfman et al., 2003; reviewed in Woolley and Schwartzkroin, 1998) and, although this area of research is controversial, appear to play roles in hippocampus-dependent learning and memory (reviewed in Asthana, 2004; Desmond and Levy, 1997; Gibbs and Gabor, 2003). The effects of estrogens are mediated in part by estrogen receptors, probably the estrogen receptor alpha, expressed in the hippocampus, including CA1 (Orikasa et al., 2000) or non-nuclear estrogen receptors (Shingo and Kito, 2005; Kuroki et al., 2000). In addition, estrogens may exert indirect effects on hippocampal spinogenesis via hormone-sensitive cholinergic or other afferents to the hippocampus (Towart et al., 2003; Lam and Leranth, 2003; Daniel and Dohanich, 2001; reviewed in Gibbs, 2000b). Thus, sex steroids, through both direct and indirect mechanisms, play a critical importance from developmental period to adulthood for organizing and reorganizing neuronal circuitry driving neuroendocrine and behavioral functions (Matsumoto, 1991).

In the present study, we examined the effects of estradiol treatment on hippocampal dendritic spines in CA1sr of pubertal rats. Although there were a number of potential approaches, we chose to evaluate OVX rats at the same age (P49) at which we studied intact rats. In this study we allowed rats to develop in the absence $(\mathrm{OVX}+\mathrm{VEH})$ or the present $(\mathrm{OVX}+\mathrm{E} 2)$ of the developmental increase in estradiol, and examined the effects on spinophilin immunoreactivity in the CA1sr. Surprisingly, there was neither an effect of OVX compared to the intact rats at 49 days (compare Figs. 3 and 5), nor was there an effect of estradiol treatment compared to vehicle treatment (compare rats within Fig. 5). Thus, the age-related decline in spinophilin immunoreactivity cannot be attributed to increasing endogenous or exogenous peripheral estradiol concentrations. Rather, there may be an estradiol-independent mechanism for maturational changes in hippocampal synaptic plasticity. Future studies will address the nature of the loss of spinophilin immunoreactivity in CA1sr during the pubertal process.

\section{Conclusions}

The peripubertal period of postnatal development is an important adaptative period, which is influenced by both steroid-dependent and steroid-independent events resulting in the formation of the future adult behavior and physiology (Romeo, 2003). This period is often associated with dramatic life changes depending upon the social dynamics of the species; for example, in some mammalian species, males or females are driven from a family group to prevent inbreeding. This life period in female mammals is also associated with the first efforts to locate and breed with conspecific mates, and shortly thereafter, to prepare for and raise offspring. These and other changes in life circumstances are best adapted to by a brain that continues to exhibit appropriate synaptic plasticity. Thus, the dendritic spine loss shown here in hippocampus may be one of the developmental changes that enables the establishment of the future stable spine number of the mature individual (Matsumoto, 1991).

\section{Acknowledgments}

This study was supported by NIH-NIA Grant PO1-AG16765 (ACG, JHM) and NSF Grant IBN-0334221 (ACG). We thank Patrick Hof, M.D., Jiandong Hao, M.D., Ph.D., Chester C. Sherwood, Ph.D. and Peter A. Needle for their technical advice and assistance.

\section{References}

Adams, M.M., Morrison, J.H., 2003. Estrogen and the aging hippocampal synapse. Cereb. Cortex 13, 1271-1275.

Adams, M.M., Shah, R.A., Janssen, W.G.M., Morrison, J.H., 2001. Different modes of hippocampal plasticity in response to estrogen in young and aged female rats. Proc. Natl. Acad. Sci. U. S. A 98, 8071-8076.

Adams, M.M., Fink, S.E., Shah, R.A., Janssen, W.G.M., Hayashi, S., Milner, T.A., McEwen, B.S., Morrison, J.H., 2002. Estrogen and aging affect the subcellular distribution of estrogen receptor alpha in the hippocampus of female rats. J. Neurosci 22, 3608-3614.

Allen, P.B., Ouimet, C.C., Greengard, P., 1997. Spinophilin, a novel protein phosphatase 1 binding protein localized to dendritic spines. Proc. Natl. Acad. Sci. U. S. A 94, 9956-9961.

Anderson, C.H., 1982. Changes in dendritic spine density in the preoptic area of the female rat at puberty. Brain Res. Bull 8, 261-265.

Andersen, S.L., Teicher, M.H., 2004. Delayed effects of early stress on hippocampal development. Neuropsychopharmacol 29, 1988-1993.

Apter, D., 1997. Development of the hypothalamic-pituitary-ovarian axis. Ann. N.Y. Acad. Sci 816, 9-21.

Asthana, S., 2004. Estrogen and cognition: a true relationship? J. Am. Geriatr. Soc. 52, 316-318.

Bi, R., Broutman, G., Foy, M.R., Thompson, R.F., Baudry, M., 2000. The tyrosine kinase and mitogen-activated protein kinase pathways mediate multiple effects of estrogen in hippocampus. Proc. Natl. Acad. Sci. U. S. A 97, 3602-3607. 
Bourgeois, J.P., Rakic, P., 1993. Changing of synaptic density in the primary visual cortex of the rhesus monkey from fetal to adult age. J. Neurosci 13, 2801-2820

Bourgeois, J.P., Goldman-Rakic, P.S., Rakic, P., 1994. Synaptogenesis in the prefrontal cortex of rhesus monkeys. Cereb. Cortex 4, 78-96.

Bjugn, R., 1993. The use of the optical disector to estimate the number of neurons, glial and endothelial cells in the spinal cord of the mouse - with a comparative note on the rat spinal cord. Brain Res 627, 25-33.

Brake, W.G., Alves, S.E., Dunlop, J.C., Lee, S.J., Bulloch, K., Allen, P.B., Greengard, P., McEwen, B.S., 2001. Novel target sites for estrogen action in the dorsal hippocampus: an examination of synaptic proteins. Endocrinology $142,1284-1289$.

Carrer, H.F., Agustin, A., 1982. Ultrastructural changes in the hypothalamic ventromedial nucleus of ovariectomized rats after estrogen treatment. Brain Res 240, 221-233.

Chakraborty, T.R., Gore, A.C., 2004. Aging-related changes in ovarian hormones, their receptors, and neuroendocrine function. Exp. Biol. Med 229, 977-987.

Chakraborty, T.R., Hof, P.R., Ng, L., Gore, A.C., 2003a. Stereologic analysis of estrogen receptor alpha $(\mathrm{ER} \alpha)$ expression in rat hypothalamus and its regulation by aging and estrogen. J. Comp. Neurol 466, 409-421.

Chakraborty, T.R., Ng, L., Gore, A.C., 2003b. Age-related changes in estrogen receptor beta in rat hypothalamus: a quantitative analysis. Endocrinology 144, 4164-4171.

Choi, J.M., Romeo, R.D., Brake, W.G., Bethea, C.L., Rosenwaks, Z., McEwen, B.S., 2003. Estradiol increases pre- and post-synaptic proteins in the CA1 region of the hippocampus in female rhesus macaques (Macaca mulatta) Endocrinology 144, 4734-4738.

Clough, R.W., Rodriguez-Sierra, J.F., 1983. Synaptic changes in the hypothalamus of the prepuberal female rat administered estrogen. Am. J. Anat 167, 205-214.

Cottrell, E.C., Campbell, R.E., Han, S.K., Herbison, A.E., 2006. Postnatal remodeling of dendritic structure and spine density in gonadotropin-releasing hormone neurons. Endocrinology 147, 3652-3661.

Daftary, S.S., Gore, A.C., 2003. Developmental changes in hypothalamic insulin-like growth factor-1: relationship to GnRH neurons. Endocrinology 144, 2034-2045.

Daniel, J.M., Dohanich, G.P., 2001. Acetylcholine mediates the estrogeninduced increase in NMDA receptor binding in CA1 of the hippocampus and the associated improvement in working memory. J. Neurosci 21, 6949-6956.

Desmond, N.L., Levy, W.B., 1997. Ovarian steroidal control of connectivity in the female hippocampus: an overview of recent experimental findings and speculations on its functional consequences. Hippocampus 7, 239-245.

Docke, F., Smollich, A., 1978. Morphological differentiation of limbic nuclei during sexual maturation of female rats. Endokrinologie 72, 1-8.

Feng, J., Yan, Z., Ferreira, A., Tomizawa, K., Liauw, J.A., Zhuo, M., Allen, P.B., Ouimet, C.C., Greengard, P., 2000. Spinophilin regulates the formation and function of dendritic spines. Proc. Natl. Acad. Sci. U. S. A 97, 9287-9292.

Frankfurt, M., Gould, E., Wolley, C.S., McEwen, B.S., 1990. Gonadal steroids modify dendritic spine density in ventromedial hypothalamic neurons: a Golgi study in the adult rat. Neuroendocrinology 51, 530-535.

Gibbs, R.B., Gabor, R., 2003. Estrogen and cognition: applying preclinical findings to clinical perspectives. J. Neurosci. Res 74, 637-643.

Gibbs, R.B., 2000a. Long-term treatment with estrogen and progesterone enhances acquisition of a spatial memory task by ovariectomized aged rats. Neurobiol. Aging 21, 107-116.

Gibbs, R.B., 2000b. Oestrogen and the cholinergic hypothesis: implications for oestrogen replacement therapy in postmenopausal women. Novartis Found. Symp 230, 94-107.

Gore, A.C., 2001. Gonadotropin-releasing hormone neurons, NMDA receptors, and their regulation by steroid hormones across the reproductive life cycle. Brain Res. Rev 37, 235-248.

Gore, A.C., Wu, T.J., Rosenberg, J.J., Roberts, J.L., 1996. Gonadotropinreleasing hormone and NMDA-R1 gene expression and colocalization change during puberty in female rats. J. Neurosci 16, 5281-5289.

Gore, A.C., Oung, T., Woller, M.J., 2002. Age-related changes in hypothalamic gonadotropin-releasing hormone $(\mathrm{GnRH})$ and NMDA receptor gene expression and their regulation by estrogen in the female rat. J. Neuroendocrinol 14, 300-309.

Gould, E., Woolley, C., Frankfurt, M., McEwen, B.S., 1990. Gonadal steroids regulate dendritic spine density in hippocampal pyramidal cells in adulthood. J. Neurosci 10, 1286-1291.

Gundersen, H.J., Bendtsen, T.F., Korbo, L., Marcussen, N., Moller, A., Nielsen, K., Nyengaard, J.R., Pakkenberg, B., Sorensen, F.B., Vesterby, A., West, M.J., 1988. Some new, simple and efficient stereological methods and their use in pathological research and diagnosis. Acta Pathol. Microbiol. Immunol. Scand 96, 379-394.

Hammond, C.B., 1996. Menopause and hormone replacement therapy: an overview. Obstet. Gynecol. 87, 2S-15S.

Hao, J., Janssen, W.G.M., Tang, Y., Roberts, J.A., McKay, H., Lasley, B., Allen, P.B., Greengard, P., Rapp, P.R., Kordower, J.H., Hof, P.R., Morrison, J.H., 2003. Estrogen increases the number of spinophilin-immunoreactive spines in the hippocampus of young and aged female rhesus monkeys. J. Comp. Neurol 465, 540-550.

Kanit, L., Taskiran, D., Yilmaz, A., Balkan, B., Demirgoren, S., Furedy, J.J., Pogun, S., 2000. Sexually dimorphic cognitive style in rats emerges after puberty. Brain Res. Bull 52, 243-248.

Komarova, N.L., Nowak, M.A., 2001. Natural selection of the critical period for language acquisition. Proc. R. Soc. Lond 268, 1189-1196.

Koshibu, K., Levitt, P., Ahrens, E.T., 2004. Sex-specific, postpuberty changes in mouse brain structures revealed by three-dimensional magnetic resonance microscopy. NeuroImage 22, 1636-1645.

Krasnoff, A., Weston, L.M., 1976. Puberal status and sex differences: activity and maze behavior in rats. Dev. Psychobiol 9, 261-269.

Kuroki, Y., Fukushima, K., Kanda, Y., Mizuno, K., Watanabe, Y., 2000. Putative membrane-bound estrogen receptors possibly stimulate mitogen-activated protein kinase in the rat hippocampus. Eur. J. Pharmacol 400, 205-209.

Lam, T.T., Leranth, C., 2003. Role of the medial septum diagonal band of Broca cholinergic neurons in oestrogen-induced spine synapse formation on hippocampal CA1 pyramidal cells of female rats. Eur. J. Neurosci 17, 1997-2005.

Luine, V.N., Jacome, L.F., MacLusky, N.J., 2003. Rapid enhancement of visual and place memory by estrogens in rats. Endocrinology 144, 2836-2844.

Matsumoto, A., 1991. Synaptogenic action of sex steroids in developing and adult neuroendocrine brain. Psychoneuroendocrinology 16, 25-40.

Matsumoto, A., Arai, Y., 1976. Developmental changes in synaptic formation in the hypothalamic arcuate nucleus of female rats. Cell Tissue Res 169, 143-156.

Matsumoto, A., Arai, Y., 1977. Precocious puberty and synaptogenesis in the hypothalamic arcuate nucleus in pregnant mare serum gonadotropin (PMSG) treated immature female rats. Brain Res 129, 375-378.

McCarthy, J.B., Milner, T.A., 2003. Dendritic ribosomes suggest local protein synthesis during estrous cynaptogenesis. Neuro. Report 14, 1357-1360.

Meyer, G., Ferres-Torres, R., Mas, M., 1978. The effects of puberty and castration on hippocampal dendritic spines of mice. A Golgi study. Brain Res 155, 108-112.

Mitra, S.W., Hoskin, E., Yudkovitz, J., Pear, L., Wilkinson, H.A., Hayashi, S., Pfaff, D.W., Ogawa, S., Rohrer, S.P., Schaeffer, J.M., McEwen, B.S., Alves, S.E., 2003. Immunolocalization of estrogen receptor $\beta$ in the mouse brain: comparison with estrogen receptor $\alpha$. Endocrinology 144, 2055-2067.

Murphy, D.D., Segal, M., 1996. Regulation of dendritic spine density in cultured rat hippocampal neurons by steroid hormones. J. Neurosci 16, 4059-4068.

Orikasa, C., McEwen, B.S., Hayashi, H., Sakuma, Y., Hayashi, S., 2000. Estrogen receptor alpha, but not beta, is expressed in the interneurons of the hippocampus in prepubertal rats: an in situ hybridization study. Dev. Brain Res 120, 245-254.

Reier, P.J., Cullen, M.J., Froelich, J.S., Rothchild, I., 1977. The ultrastructure of the developing medial preoptic nucleus in the postnatal rat. Brain Res 122, 415-436.

Romeo, R.D., 2003. Puberty: a period of both organizational and activational effects of steroid hormones on neurobehavioural development. J. Neuroendocrinol 15, 1185-1192.

Safranski, T.J., Lamberson, W.R., Keisler, D.H., 1993. Correlations among three measures of puberty in mice and relationships with estradiol concentration and ovulation. Biol. Reprod 48, 669-673.

Schenk, F., 1985. Development of place navigation in rats from weaning to puberty. Behav. Neural Biol 43, 69-85. 
Segarra, A., McEwen, B.S., 1991. Estrogen increases spine density in ventromedial hypothalamic neurons of peripubertal rats. Neuroendocrinology $54,365-372$.

Scharfman, H.E., Mercurio, T.C., Goodman, J.H., Wilson, M.A., MacLusky, N.J., 2003. Hippocampal excitability increases during the estrous cycle in the rat: a potential role for brain-derived neurotrophic factor. J. Neurosci 23, $11641-11652$.

Shingo, A.S., Kito, S., 2005. Estradiol induces PKA activation through the putative membrane receptor in the living hippocampal neuron. J. Neural Transm 112, 1469-1473.

Tang, Y., Janssen, W.G.M., Hao, J., Roberts, J.A., McKay, H., Lasley, B., Allen, P.B., Greengard, P., Rapp, P.R., Kordower, J.H., Hof, P.R., Morrison, J.H., 2004. Estrogen replacement increases spinophilin-immunoreactive spine number in the prefrontal cortex of female rhesus monkeys. Cereb. Cortex 14, 215-223.

Towart, L.A., Alves, S.E., Znamensky, V., Hayashi, S., McEwen, B.S., Milner, T.A., 2003. Subcellular relationships between cholinergic terminals and estrogen receptor-alpha in the dorsal hippocampus. J. Comp. Neurol 463, 390-401.

Van Lookeren Campagne, M., Oestreicher, B.A., Van Der Krift, T.P., Gispen, W.H., Verkleij, A.J., 1991. Freeze-substitution and Lowicryl HM20 embedding of fixed rat brain: suitability for immunogold ultrastructural localization of neural antigens. J. Histochem. Cytochem 39, 1267-1279.

Warren, S.G., Humphreys, A.G., Juraska, J.M., Greenough, W.T., 1995. LTP varies across the estrous cycle: enhanced synaptic plasticity in proestrus rats. Brain Res 703, 26-30.

West, M.J., Slomianka, L., Gundersen, H.J., 1991. Unbiased stereological estimation of the total number of neurons in the subdivisions of the rat hippocampus using the optical fractionator. Anat. Rec 231, 482-497.
Woo, T.U., Pucak, M.L., Kye, C.H., Matus, C.V., Lewis, D.A., 1997. Peripubertal refinement of the intrinsic and associational circuitry in monkey prefrontal cortex. Neuroscience 80, 1149-1158.

Woolley, C.S., McEwen, B.S., 1992. Estradiol mediates fluctuation in hippocampal synapse density during the estrous cycle in the adult rat. J. Neurosci 12, 2549-2554.

Woolley, C.S., McEwen, B.S., 1994. Estradiol regulates hippocampal dendritic spine density via an $\mathrm{N}$-methyl-D-aspartate receptor-dependent mechanism. J. Neurosci 14, 7680-7687.

Woolley, C.S., Schwartzkroin, P.A., 1998. Hormonal effects on the brain. Epilepsia 39 (Suppl 8), S2-S8.

Woolley, C.S., Gould, E., Frankfurt, M., McEwen, B.S., 1990. Naturally occurring fluctuation in dendritic spine density on adult hippocampal pyramidal neurons. J. Neurosci 10, 4035-4039.

Woolley, C.S., Wenzel, H.J., Schwartzkroin, P.A., 1996. Estradiol increases the frequency of multiple synapse boutons in the hippocampal CA1 region of the adult female rat. J. Comp. Neurol 373, 108-117.

Wright, B.A., Zecker, S.G., 2004. Learning problems, delayed development, and puberty. Proc. Natl. Acad. Sci. U. S. A 101, 9942-9946.

Yun, A.J., Bazar, K.A., Lee, P.Y., 2004. Pineal attrition, loss of cognitive and onset of puberty during the teen years: is it a modern maladaptation exposed by evolutionary displacement? Med. Hypothesis 63, 939-950.

Zecevic, N., Rakic, P., 1991. Synaptogenesis in monkey somatosensory cortex. Cereb. Cortex 1, 510-523.

Zehr, J.L., Todd, B.J., Schulz, K.M., McCarthy, M.M., Sisk, C.L., 2006. Dendritic pruning of the medial amygdala during pubertal development of the male Syrian hamster. J. Neurobiol 66, 578-590. 